\title{
Massive star clusters in high-redshift star-forming galaxies seen at a 100 pc scale thanks to strong gravitational lensing
}

\author{
Miroslava Dessauges-Zavadsky ${ }^{1}$, Antonio Cava ${ }^{1}$, \\ Valentina Tamburello ${ }^{2}$, Daniel Schaerer ${ }^{1}$, Lucio Mayer ${ }^{2}$, \\ Johan Richard ${ }^{3}$ and Pablo G. Pérez González ${ }^{4}$ \\ ${ }^{1}$ Observatoire de Genève, Université de Genève, \\ 51 Chemin des Maillettes, 1290 Versoix, Switzerland \\ ${ }^{2}$ Center for Theoretical Astrophysics and Cosmology, Institute for Computational Science, \\ University of Zurich, Winterthurerstrasse 190, 8057 Zurich, Switzerland \\ ${ }^{3}$ CRAL, Observatoire de Lyon, Université Lyon 1, \\ 9 Avenue Ch. André, 69561 Saint Genis Laval Cedex, France \\ ${ }^{4}$ Departamento de Astrofísica y Ciencias de la Atmósfera, Facultad de CC. Físicas, \\ Universidad Complutense de Madrid, 28040 Madrid, Spain
}

\begin{abstract}
High-resolution imaging reveals clumpy morphologies among $z=1-3$ galaxies. Most of these galaxies are dominated by disk rotation, which led to conclude that the observed clumps are generated from disk fragmentation due to gravitational instability. Despite the kpcscale resolution attained by the most advanced facilities and numerical simulations, these clumps are barely resolved at $z>1$. Thanks to the stretching and magnification power provided by gravitational lensing, we reach the sub-kpc resolving power to unveil their physics. From our literature compilation of data, we show that without lensing there is a bias toward clumps with high masses and sizes. The high-redshift clumps identified in lensed galaxies have stellar masses 2 orders of magnitude lower and a median size of 250 pc. They resemble local star clusters observed in the most intensively star-forming galaxies. The clump masses and sizes observed in lensed galaxies agree with new simulations, which show that the Toomre instability criterion overestimates the clump masses by a factor of $5-6$.
\end{abstract}

Keywords. Gravitational lensing, galaxies: evolution, galaxies: high-redshift

\section{Introduction}

Deep rest-frame UV HST observations show that $z=1-3$ galaxies, at the peak of the cosmic star formation activity, do not follow the classical Hubble classification: they are mostly irregular and clumpy. Six morphological types have been proposed, among which 5 show prominent clumps or massive star clusters (Elmegreen et al. 2005, 2009). While these clumps have been initially associated with interactions and mergers, evidence has been accumulating that only a minority of high-redshift galaxies (about 30\%) are in the process of merging. Kinematical galactic internal structure suggests that a substantial fraction of $z \sim 1-2$ star-forming galaxies, even those with clumpy morphologies, are dominated by ordered disk rotation, as observationally constrained by $\mathrm{H} \alpha$ line IFU spectroscopy (Genzel et al. 2006; Förster Schreiber et al. 2009; Wisnioski et al. 2015).

These high-redshift disks are very different from their counterparts in the local Universe. They are characterized by high velocity dispersions, low rotation velocity to dispersion ratios, high molecular gas fractions, and high star formation rates (e.g., Wisnioski et al. 2015; Dessauges-Zavadsky et al. 2015; Schreiber et al. 2015). These disks thus 
Table 1. Physical properties of simulated clumps.

\begin{tabular}{lcc}
\hline & $\begin{array}{c}\text { Recent simulations by } \\
\text { Tamburello } \text { et al. }(2015)\end{array}$ & $\begin{array}{c}\text { Previous simulations by } \\
\text { Ceverino } \text { et al. } 2010\end{array}$ \\
\hline Clump sizes $(r)$ & $100-250 \mathrm{pc}$ & $0.5-1 \mathrm{kpc}$ \\
Stellar masses $\left(M_{* \text { clump }}\right)$ & $10^{7}-10^{8} \mathrm{M} \odot$ & $10^{8}-10^{9} \mathrm{M} \odot$ \\
Contribution to the bulge & Negligible & Significant \\
\hline
\end{tabular}

appear to be highly turbulent, thick, gas-rich, and strongly star-forming. In this context, the dominant interpretation of the origin of clumps, as gravitationally bound structures, is their production by disk fragmentation resulting from gravitational instability. It is debated whether, due to clump interactions and dynamical friction against the surrounding disk, clumps migrate toward the centre of their host galaxy and coalesce into a young bulge, or are disrupted by tidal forces or stellar feedback to form part of a thick disk.

\section{New prescriptions for the gravitational fragmentation}

The gravitational fragmentation of gas-rich, turbulent galactic disks into giant clumps has been addressed by various numerical simulations (e.g., Bournaud et al. 2009; Dekel et al. 2009; Ceverino et al. 2010). Tamburello et al. (2015, hereafter T15) revisit the large-scale fragmentation of galactic disks. Their simulations study isolated galaxies with a wide range of stellar masses, $M_{*} \sim 10^{10}-10^{11} \mathrm{M}_{\odot}$, and initial conditions based on the ARGO cosmological hydrodynamical simulations. Their mass resolution is as high as $10^{4} \mathrm{M}_{\odot}$ and they use an efficient stellar feedback model that has been shown to produce realistic spiral galaxies at low redshift, with stellar masses in agreement with constraints (Guedes et al. 2011). They investigate, in parallel, the Toomre, Jeans, and Boley et al. (2010) gravitational instability criteria. Their main findings are that the clumps that result from fragmentation using the Boley et al. instability criterion are smaller both in size and mass (see Table1). The Toomre mass overestimates the clump masses by a factor of $5-6$. Some clumps do still reach a stellar mass as large as $M_{* \text { clump }} \simeq 10^{9} \mathrm{M}_{\odot}$ 0.5 Gyr after formation, but those clumps result from multiple clump-clump mergers and do not reflect the mass scale set by fragmentation (see also Madelker et al. in prep.). The largest clumps are found in the most massive galaxies, because these galaxies produce more small clumps and host more mergers that can boost the individual clump mass.

\section{Observational constraints on high-redshift clumps}

Two approaches have been used to obtain observational constraints on $z>1$ clumps. The first approach focusses on the study of field galaxies with high-resolution HST imaging and adaptive optic IFU spectroscopy. This approach has the sensitivity as a first limitation, which implies a bias toward galaxies with high star formation rates (SFR) and hence physical conditions not observed in the local Universe. A second limitation is the spatial resolution, in general larger than $1 \mathrm{kpc}$ at $z>1$ (Genzel et al. 2011; Förster Schreiber et al. 2011; Guo et al. 2012; Wisnioski et al. 2012; Swinbank et al. 2012). The second approach aims to study gravitationally lensed galaxies. This approach has the following advantage. Strong lensing leads to the stretching of the source image, that conserving the surface brightness produces an amplification in the total observed flux proportional to the stretching factor. This implies the release of the sensitivity limit and enables to study fainter galaxies with lower SFR. More importantly, it also releases the limit on the spatial resolution, such that sub-kpc scales down to $100 \mathrm{pc}$ become reachable (Jones et al. 2010; Livermore et al. 2012, 2015; Adamo et al. 2013; Wuyts et al. 2014). For 

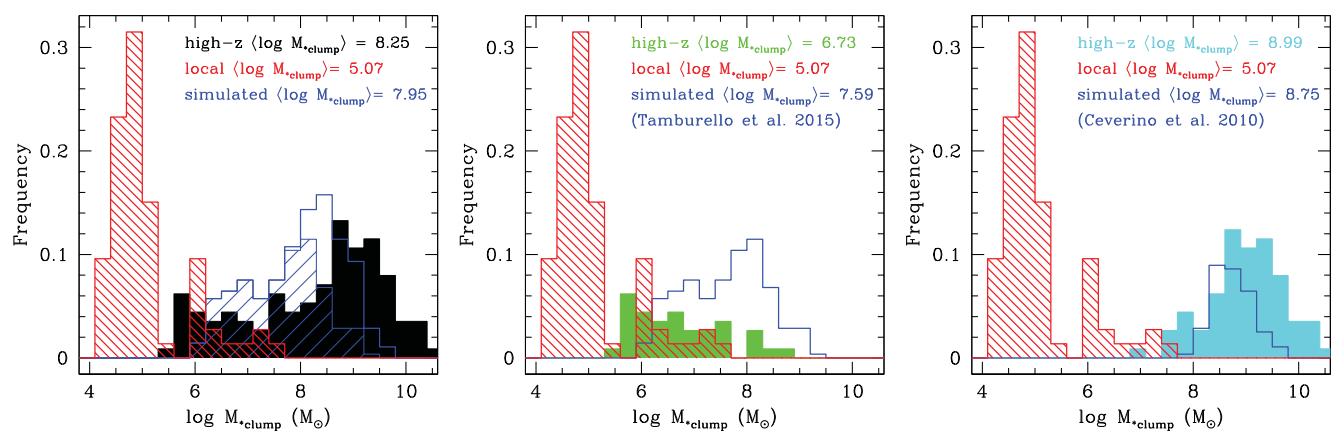

Figure 1. Left: Histograms of the stellar mass distribution of high-redshift clumps (filled black), local star clusters (hatched red), and simulated clumps (open blue for Ceverino et al. (2010) and hatched blue for T15). Middle: Same, but for the high-redshift clumps in "lensed" galaxies (filled green). Right: Same, but for the high-redshift clumps in "field" galaxies (filled cyan).

both field and lensed galaxies, the common physical parameters derived for high-redshift clumps are: their size, distance to the centre, stellar mass, SFR, and age.

\subsection{Stellar mass distribution}

In Fig. 1 we compare the stellar mass distributions of high-redshift clumps, local star clusters, and simulated clumps (using our compilation from the literature, exhaustive for $z>1$ clumps). As shown in the left panel, the high-redshift clumps have on average a factor of 1000 larger masses than local cluster complexes, whereas their masses globally are in line with the clump masses predicted by simulations, except for the higher mass end. The observed high- $M_{* \text { clump }}$ peak is not even reproduced by the T15 simulations. Interestingly, if we plot the clumps identified in lensed galaxies and those studied in field galaxies separately (middle and right panels), we observe that their stellar mass distributions are clearly different, with their means $\left\langle M_{* \text { clump }}\right\rangle_{\text {lensed }}=5.4 \times 10^{6} \mathrm{M}_{\odot}$ and $\left\langle M_{* \text { clump }}\right\rangle_{\text {field }}=9.8 \times 10^{8} \mathrm{M}_{\odot}$ diverging by 2 orders of magnitude. The $M_{* \text { clump }}$ in lensed galaxies reach the low mass domain found in the recent T15 simulationst. This is not the case of the $M_{* \text { clump }}$ in field galaxies that better agree with the older Ceverino et al. (2010) simulations. This shows a clear bias toward the high $M_{* \text { clump }}$ in field galaxies. As a result, the physical properties of clumps as measured in field galaxies may not be representative of the intrinsic properties of high-redshift star clusters because of possible blends of clumps due to the scarce spatial resolution. When spatially resolving the clumps in lensed galaxies, they still have a factor of 100 larger masses than the bulk of local star cluster complexes, but they resemble complexes in the most intensively star-forming galaxies in the local Universe, like the Antennae and NGC 6946.

\subsection{Scaling relations: $M_{* \text { clump }}$ - size and $S F R_{\text {clump }}-$ size}

In the local galaxies, the stellar mass of the star cluster complexes is found to scale with their size: $M_{* \text { clump }} \propto r^{2}$, in a similar way as for the giant molecular clouds (GMCs) (Bastian et al. 2005; Bolatto et al. 2008). This relation suggests a constant gas surface density. In Fig. 2 we show the stellar masses of high-redshift clumps, local star clusters, and simulated clumps as a function of their sizes. Interestingly, most clumps analysed in lensed galaxies follow the $M_{* \text { clump }}$ - size relation of local GMCs. This suggests that their formation process might be very close to the hierarchical process that governs the formation of cluster complexes in the local Universe. On the other hand, all the other

$\dagger$ The high mass domain is under-represented, because lensing mostly selects galaxies in the lower end of the stellar mass distribution, while in T15 there is the same number of runs with massive and moderate galaxies. 


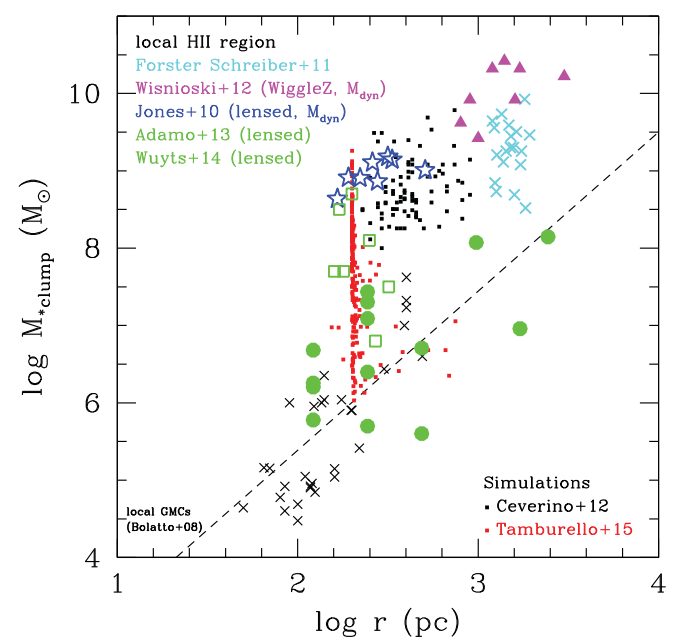

Figure 2. Clump stellar masses as a function of their sizes.

high-redshift clumps observed in field galaxies sit above this relation. This could be due first to the spatial resolution effects, as discussed in Sect. 3.1, that blend clumps to fewer and more massive. The second explanation could be that galaxies at $z>2$ have disks with larger gas surface densities capable of forming more massive clumps, an effect to be combined with the detection limits that also favour the selection of more massive host galaxies with clumps forming in more extreme environments. This may induce a vertical shift of the $M_{* \text { clump }}$ - size relation for the high-redshift clumps (see Adamo et al. 2013).

This shift is observed in the $\mathrm{SFR}_{\text {clump }}$ - size relation, the second scaling relation of local star clusters: $\mathrm{SFR}_{\text {clump }} \propto r^{2}$, which suggests a constant surface brightness. When comparing the high-redshift star clusters with the local ones, we observe that the former have surface brightnesses up to 100 times higher than the latter. The difference is too strong to be fully explained by resolution and sensitivity effects. Livermore et al. (2012, 2015) suggested that the clump surface brightness or the $\mathrm{SFR}_{\text {clump }}$ - size relation evolves with redshift. They built up samples of $\mathrm{H} \alpha$ luminosity measurements for clumps in lensed galaxies for three redshift intervals $(1<z<1.5,1.5<z<3, z>3)$, and find that the characteristic luminosity of the clump luminosity function clearly increases with redshift.

\subsection{Contribution of clumps to host galaxies}

When analysing the contribution in stellar mass of individual clumps to the host galaxy, we find that it ranges from $<1 \%$ to $50 \%$. The upper end of this contribution is certainly overestimated, since in the field galaxies the observed clumps very likely are blends of several individual clumps. The T15 simulations indeed predict a maximum contribution in stellar mass of the individual clumps to the host of at most $20 \%$. The contribution of the sum of all clumps to stellar mass of the host peaks at $\sim 20 \%$, in very good agreement with the T15 simulations. These numbers show that the contribution in stellar mass of both the individual clumps and the sum of all clumps in one host galaxy is relatively small to have an important impact on the bulge growth.

\subsection{Clump survival, growth, and migration}

Since the first observations of clumps in high-redshift star-forming galaxies, the fate of these clumps was questioned. Two scenarios were initially invoked: either the clumps are disrupted by stellar feedback and tidal torques, or the clumps migrate toward the galactic centre of their host galaxies due to interactions and dynamical friction. When 

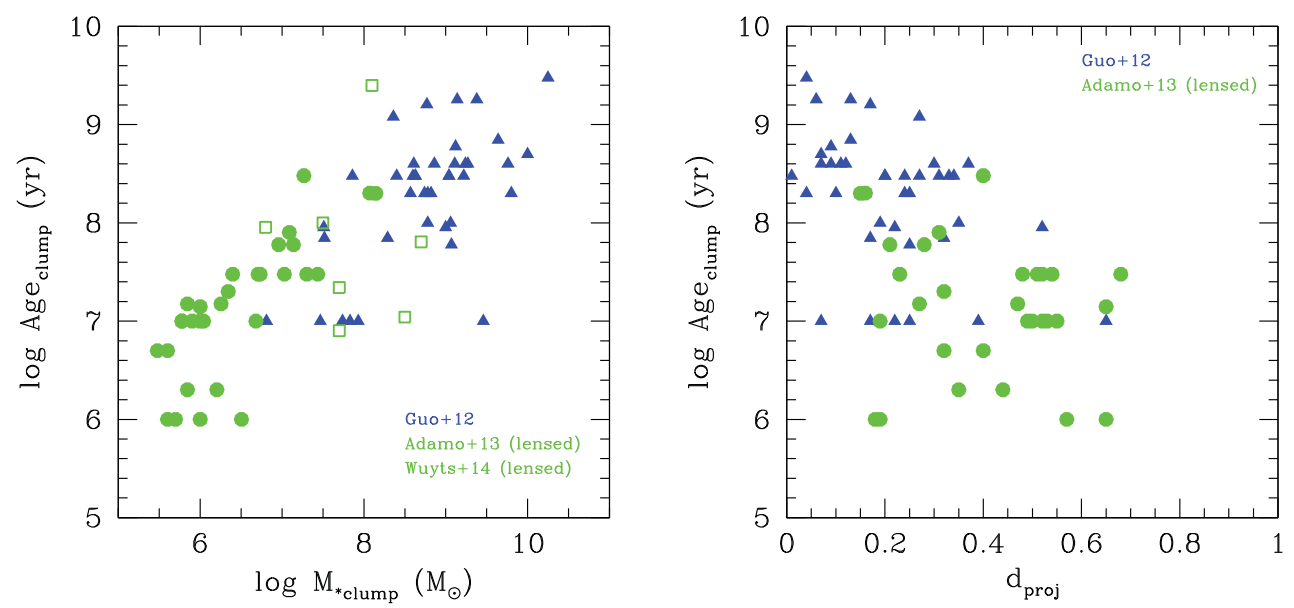

Figure 3. Left: Clump ages as a function of their stellar masses. Right: Clump ages as a function of their projected distance to the galactic centre of the host galaxies.

estimating the clump ages from the SED modeling, we observe that clumps may reach ages up to $0.5-1$ Gyr and hence survive over a long timescale. In the left panel of Fig. 3, we show the clump ages as a function of their stellar masses and find that the older clumps are in fact the most massive ones. This agrees with the T15 simulations, where they show that $M_{* \text { clump }}$ grows with time because of clump-clump mergers. Starting with a mean $\left\langle M_{* \text { clump }}\right\rangle \simeq 5 \times 10^{7} \mathrm{M}_{\odot}$ at the time of the clump formation, a mean $\left\langle M_{* \text { clump }}\right\rangle \simeq 5 \times 10^{8} \mathrm{M}_{\odot}$ is reached $\sim 0.5$ Gyr after formation (see Fig. 4 ).

In the right panel of Fig. 3, we show the clump ages as a function of their projected distances to the centre of the host galaxies. One observes a trend for the older clumps to be the closer to the galactic centres. This suggests that clumps survive long enough to migrate toward the centre of the hosts. Nevertheless, according to T15 and as seen in Sect. 3.3, these clumps only have a marginal contribution on the galactic bulge growth.

\section{Conclusions}

It has been shown that a large fraction of star-forming galaxies at $z=1-3$ are found to be disks in rotation. These high-redshift disks are highly turbulent, thick, gas-rich, and strongly star-forming. They induce the formation of clumps or star clusters by disk fragmentation as a result of the gravitational instability. Do these clumps resemble the star cluster complexes observed in the Milky Way and local galaxies?

When analysing the physical properties of the high-redshift clumps observed in field galaxies and the clumps spatially resolved in gravitationally lensed galaxies separately, we find that the stellar masses and sizes of the former are significantly larger than those of local star clusters. On the other hand, the high-redshift clumps in lensed galaxies, although they still have larger stellar masses and sizes, with a mean $\left\langle M_{* \text { clump }}\right\rangle=5.4 \times$ $10^{6} \mathrm{M}_{\odot}$ and a median $r=250 \mathrm{pc}$, relative to the bulk of local star clusters, resemble the star complexes in the Antennae and NGC 6946. These results show evidence for a bias toward clumps with high $M_{* \text { clump }}$ and high sizes in field galaxies due to instrumental spatial resolution limitations that blend clumps to fewer and more massive clumps.

Recent simulations by T15 revisit the gravitational fragmentation of turbulent, gas-rich galactic disks and predict smaller clumps in both stellar mass and size. Their findings are in agreement with the physical properties of clumps observed in lensed galaxies. 

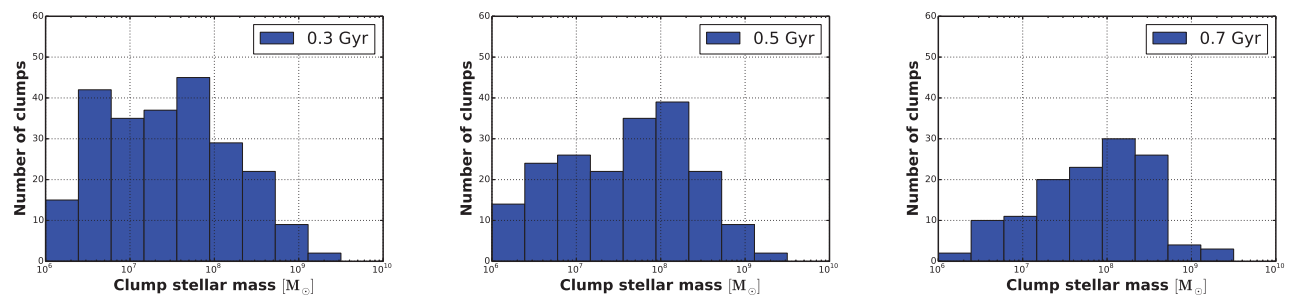

Figure 4. Histograms of the clump stellar mass distribution as a function of time, as derived in the T15 simulations.

They show that the Toomre gravitational instability criterion, so far mostly considered in simulations, overestimates the clump masses by a factor of $5-6$.

The scaling relations $M_{* \text { clump }}$ - size and $\mathrm{SFR}_{\text {clump }}$ - size empirically determined in star cluster complexes of local galaxies seem to hold for clumps at high redshift, but with a vertical shift well established in the $\mathrm{SFR}_{\text {clump }}$ - size relation and to be confirmed in the $M_{* \text { clump }}$ - size relation. The stellar mass of high-redshift clumps grows with time due to multiple clump-clump mergers. The high-redshift clumps may survive as long as $1 \mathrm{Gyr}$ and migrate toward the galactic centre of their host galaxies. Nevertheless, the clump contribution to the bulge build-up is negligible, with the mean contribution of the sum of all clumps representing $\sim 20 \%$ of the total stellar mass of the host galaxy.

\section{References}

Adamo, A., Östlin, G., Bastian, N., et al. 2013,ApJ, 766, 105

Bastian, N., Gieles, M., Efremov, Y. N., et al. 2005, A\&A, 443, 79

Bolatto, A. D., Leroy, A. K., Rosolowsky, E., et al. 2008, ApJ, 686, 948

Boley, A. C., Hayfield, T., Mayer, L., et al. 2010, Icarus, 207, 509

Bournaud, F., Elmegreen, B. G., \& Martig, M. 2009, ApJ, 707, L1

Ceverino, D., Dekel, A., \& Bournaud, F. 2010, MNRAS, 404, 2151

Dekel, A., Sari, R., \& Ceverino, D. 2009, ApJ, 703, 785

Dessauges-Zavadsky, M., Zamojski, M., Schaerer, D., et al.\} 2015, A\&A, 577, 50

Elmegreen, D. M., Elmegreen, B. G., Rubin, D. S., et al. 2005, ApJ, 631, 85

Elmegreen, B. G., Elmegreen, D. M., Fernandez, M. X., et al. 2009, ApJ, 692, 12

Förster Schreiber, N. M., Genzel, R., Bouché, N., et al. 2009, ApJ, 706, 1364

Förster Schreiber, N. M., Shapley, A. E., Genzel, R., et al. 2011, ApJ, 739, 45

Genzel, R., Tacconi, L. J., Eisenhauer, F., et al. 2006, Nature, 442, 786

Genzel, R., Newman, S., Jones, T., et al. 2011, ApJ, 733, 101

Guedes, J., Callegari, S., Madau, P., et al. 2011, ApJ, 742, 76

Guo, Y., Giavalisco, M., Ferguson, H. C., et al. 2012, ApJ, 757, 120

Jones, T. A., Swinbank, A. M., Ellis, R. S., et al. 2010, MNRAS, 404, 1247

Livermore, R. C., Jones, T., Richard, J., et al. 2012, MNRAS, 427, 688

Livermore, R. C., Jones, T. A., Richard, J., et al. 2015, MNRAS, 450, 1812

Schreiber, C., Pannella, M., Elbaz, D., et al. 2015, A\&A, 575, 74

Swinbank, M., Smail, I., Sobral, D., et al. 2012, ApJ, 760, 131

Tamburello, V., Mayer, L., Shen, S., et al. 2015, MNRAS, 453, 2490

Wisnioski, E., Glazebrook, K., Blake, C., et al. 2012, MNRAS, 422, 3339

Wisnioski, E., Förster Schreiber, N. M., Wuyts, S., et al. 2015, ApJ, 799, 209

Wuyts, E., Rigby, J. R., Gladders, M. D., et al. 2014, ApJ, 781, 61 